special articles

\title{
Service innovation: development of a primary care-based assessment service
}

The care of those with mental illness has moved away from asylums and in-patient care facilities. Community mental health teams (CMHTs) now deliver care but these vary widely in terms of personnel and function (Simmonds et al, 2001). Evidence suggests that CMHT management is not inferior to non-team standard care in any important respect, and that it may even be superior in promoting greater acceptance of treatment, reducing hospital admission and avoiding death by suicide (Tyrer et $a l, 2000$ ). Despite this apparent improvement, there are problems for the CMHTs and the general practitioners (GPs) who refer individuals. CMHTs are often overstretched, with large workloads, increasing amounts of administration and lack of resources, leading to concerns that individuals with more severe illness may be neglected (Harrison, 2000). Various initiatives have been undertaken to combat these problems, including forging stronger links between the CMHT and GPs, by having an on-site mental health worker (Hamilton et al, 2002; Bower \& Sibbald, 2003).

The Limavady CMHT serves a catchment area of 32000 people in a rural area of Northern Ireland. It is a traditional CMHT which provides a comprehensive general adult service. In January 2001 the team reviewed the service through a health board-led project entitled 'Improving Mental Health Services'. The aim was to examine local need and how it was being met both in primary and secondary care. The project involved members of the CMHT, GPs, service users and health board representatives, and facilitated a meaningful dialogue among the key stakeholders in relation to mental healthcare provision.

Problems consistent with those described in the literature were evident at both levels of care. Primary care services identified the high demand both in terms of numbers of individuals and the amount of time required to deal with them. Primary care staff referred all problems outside their level of expertise to the CMHT. There was a lack of knowledge and a lack of confidence in alternative statutory and non-statutory services.

For secondary services, the referral rate was rising and discharge rates were low. As a result, the waiting time for a routine new assessment could be as long as 8 months. Staff stress questionnaires identified quality of care delivered to patients and professional doubt as sources of stress. There was concern that the multifunctional character of the team led to lack of focus and the low discharge rate. This was recognised as a problem for both patients and staff. A number of initiatives emerged from the discussion. This paper focuses on one aspect, the development of a new assessment service.

\section{Development of the new service}

Until 2000, the CMHT provided multidisciplinary assessments for all individuals referred to the team. The assessment involved the consultant psychiatrist and another team member from a nursing or social work background. The assessment was a comprehensive but blanket approach to all GP referrals, irrespective of severity of the presenting problem. The system depended on the availability of a consultant and long waiting times were inevitable. A disproportionate amount of team time was spent on assessment, with less focus on active case management and discharge when appropriate.

A new post, entitled 'primary care facilitator' was piloted, where a single team member was responsible for assessment. This primary care facilitator would receive all non-emergency referrals and perform the initial assessment at the local health centres, while remaining a member of the CMHT. The facilitator would attend weekly team meetings to discuss assessments and to refer individuals to the team. There was also scope to develop mental health skills and mental health promotion strategies at the primary care level. The waiting list was cleared before the new service was implemented, so there was no inherited backlog. The post became operational in November 2001.

\section{Method}

The impact of the new post was evaluated in 2003. Quantitative data included GP referral rates, nonattendance rates, referral to the $\mathrm{CMHT}$ and discharge from the CMHT. Questionnaires were sent to 100 randomly selected patients assessed by the new primary care facilitator to gauge their satisfaction. The views of local GPs were also sought by postal questionnaire. Data were analysed with the Statistical Package for the Social Sciences version 10 .

\section{Results}

The number of routine referrals to mental health services in Limavady was slightly increased in 2002 compared with 2000 (Table 1). There was a highly significant decrease in mean waiting time from date of referral to date of first appointment, 65 days in 2000 compared with 16 days in 2002 ( $t=16.849$, d.f. $=691, P<0.001)$. This was also reflected in the percentage of patients offered an appointment within a month, 39\% in 2000 compared with $96 \%$ in $2002\left(\chi^{2}=266.8\right.$, d.f. $\left.=1, P<0.001\right)$.

Rates of non-attendance were unchanged. Outcome following assessment was significantly altered in 2002 
(Fisher's exact test $=79.68, P<0.001$ ) (Table 2 ), with more individuals being referred back to their GP (42\% v. 19\%), and more being referred to other agencies ( $9 \%$ v. $0 \%$ ). As a consequence, referrals to the CMHT dropped from $78 \%$ to $46 \%$.

\section{Views of stakeholders}

\section{Patient satisfaction}

Questionnaires were sent to 100 randomly selected patients assessed in 2002, and of these, 38 were returned, $10(26 \%)$ by men and $28(74 \%)$ by women. Thirty-three patients (87\%) felt they had been given enough information prior to their appointment; 36 (95\%) were satisfied with the length of time they had to wait for an appointment; 37 (97\%) were happy with the location of their assessment.

Twenty-nine patients (76\%) found the assessment procedure positive, with only one negative experience. The positive respondents commented on the cathartic nature of the interview; 25 out of 34 (73\%) found followup to the initial appointment positive and 7 out of 34 (21\%) found follow-up negative. Those who responded positively commented on the short time, the positive relationship with the team member and the positive outcomes. Negative comments came from those who felt they needed more intensive treatment.

\section{GP satisfaction}

Out of 22 GPs, 19 (86\%) replied to the postal questionnaire; $15(79 \%)$ felt the new assessment process had

Table 1. Referrals and appointments

\begin{tabular}{|lrc}
\hline & 2000 & 2002 \\
\hline Number of GP referrals & 349 & 368 \\
Mean waiting time for appointment (days) & 65 & $16^{*}$ \\
Patients offered appointment within & 39 & $96^{*}$ \\
1 month (\%) & & \\
Non attendance (\%) & 31.5 & 31.3 \\
\hline
\end{tabular}

$G P$, general practitioner.

${ }^{*} P<0.001$.

\begin{tabular}{|lcc|}
\hline Table 2. Outcome of assessment & & \\
\hline Outcome & 2000 & 2002 \\
& $n(\%)$ & $n(\%)$ \\
\hline Referral to CMHT & $186(78)$ & $116(46)$ \\
Discharge to GP & $45(19)$ & $107(42)$ \\
Referral to external agency & $1(0)$ & $22(9)$ \\
Admission & $7(3)$ & $0(0)$ \\
Review by nurse facilitator & $0(0)$ & $8(3)$ \\
Total & 239 & 253
\end{tabular}

$\mathrm{CMHT}$, community mental health team; GP, general practitioner.

Fisher's exact test $=79.68, P<0.001$. improved access of patients to mental health services; $12(63 \%)$ felt it had improved GP access to secondary services; 13 (68\%) thought it had improved communication between primary and secondary services; 10 (53\%) felt it had improved knowledge and understanding of secondary services in primary care; $13(68 \%)$ felt the new process had improved confidence in management of mental health difficulties in primary care.

\section{Discussion}

The introduction of the primary care facilitator to the Limavady CMHT has had a positive impact on the delivery of mental health services. General practitioners were pleased with the new system, agreeing that it improved accessibility and liaison. Patients were satisfied with the assessment process and follow-up. No comment can be made about patient preference in the absence of data regarding satisfaction with the previous system. Referral to the CMHT was significantly reduced.

The elements of this model which contributed to the success of the project need to be considered if it is to be replicated elsewhere. Local GPs were closely involved, ensuring good communication and a joint ownership of the project. The CMHT was well established, with a low staff turnover. The team was motivated to change and had strong links with the project development. The CMHT structure included a fully integrated psychologist, cognitive-behavioural therapist and psychiatrist, ensuring quick and easy referral by the facilitator when appropriate.

Any new post needs to be considered in terms of long-term sustainability. This post was designed to focus on assessment only. Absence of a therapeutic case-load decreased the clinical burden and allowed the system to offer timely appointments consistently. The primary care facilitator benefited from CMHT support, with both easy access to skills for individual case management and to professional supervision. The non-clinical component of the post will be essential to minimise burnout.

The strength of this model is that it recognises the wide range and large volume of mental health problems dealt with in primary care. The model attempts to offer GPs a flexible supportive service with intrinsic educational opportunities, without depleting secondary care services and resources for those with serious enduring mental illness.

\section{Acknowledgements}

We wish to thank the project team for offering the opportunity for change and the staff of Limavady CMHT for its successful delivery of a new service.

\section{Declaration of interest}

None. $\underset{\text { special }}{\mid} \mid$ articles 
8

special articles

\section{References}

BOWER, P. \& SIBBALD, B. (2003) On-site SIMMONDS, S., COID, J., JOSEPH, P., et mental health workers in primary care: al (2001) Community mental health effects on professional practice team management in severe mental (Cochrane Review). InThe Cochrane illness: a systematic review. British Library, issue 4. Chichester: Wiley. Journal of Psychiatry, 178, 497-502.

HAMILTON, R., GORDON, P. \& NAJl, S. TYRER, P., COID, J., SIMMONDS, S., et al (2002) Service innovation: the first year (2000) Community mental health of a brief psychiatric screening clinic in teams (CMHTs) for people with severe primary care. Psychiatric Bulletin, 26 $218-221$.

HARRISON, J. (2000) Prioritising referrals to a community mental health mental illnesses and disordered personality (Cochrane Review). InThe Cochrane Library, issue 4. Chichester: team. British Journal of General Practice, 50, 194-198.

John Brady Specialist Registrar in General Adult Psychiatry, * *Catherine McDonnell Consultant in General Adult Psychiatry, Limavady CMHT, RoeValley Hospital, Limavady BT49 0AQ, UK, e-mail: cmcdonnell@foyleby.n-i.nhs.uk 